University of South Carolina

Scholar Commons

6-15-1990

\title{
Universal scaling of nonlocal and local resistance fluctuations in small wires
}

H. Haucke

S. Washburn

A. D. Benoit

C. P. Umbach

Richard A. Webb

University of South Carolina - Columbia, webbra@mailbox.sc.edu

Follow this and additional works at: https://scholarcommons.sc.edu/phys_facpub

Part of the Physics Commons

\section{Publication Info}

Published in Physical Review B, Volume 41, Issue 18, 1990, pages 12454-12461.

Haucke, H., Washburn, S., Benoit, A.D., Umbach, C.P., and Webb, R.A. (1990). Universal scaling of nonlocal and local resistance fluctuations in small wires. Physical Review B, 41(18), 12454-12461. doi: 10.1103/

PhysRevB.41.12454

(C) 1990 The American Physical Society.

This Article is brought to you by the Physics and Astronomy, Department of at Scholar Commons. It has been accepted for inclusion in Faculty Publications by an authorized administrator of Scholar Commons. For more information, please contact digres@mailbox.sc.edu. 


\title{
Universal scaling of nonlocal and local resistance fluctuations in small wires
}

\author{
H. Haucke, ${ }^{*}$ S. Washburn, A. D. Benoit, ${ }^{\dagger}$ C. P. Umbach, and R. A. Webb \\ IBM Research Division, Thomas J. Watson Research Center, P.O. Box 218, Yorktown Heights, New York 10598
}

(Received 31 January 1990)

\begin{abstract}
Resistance fluctuations in small metal samples result from coherent transport of the carriers. The wave functions of the carriers extend into regions which are not accessible classically. We have directly measured the length dependence of the nonlocal magnetoresistance fluctuations in $\mathrm{Sb}$ and Au wires by studying regions of our samples separated from the classical current path by a distance $L$ which varied from $3 L_{\varphi}$ down to $0.2 L_{\varphi}$ (where $L_{\varphi}$ is the phase coherence length for the carriers in the metal). These fluctuations decay exponentially with $L / L_{\varphi}$. Measurements along the classical current paths scale more slowly with $L / L_{\varphi}$ than predicted by the analytical theory but are in agreement with numerical simulations. We have also studied the length dependence of the magnetic field correlation scale $B_{C}$, and we find that it is in qualitative agreement with a recent model calculation that accounts for the voltage probes.
\end{abstract}

Since the first experiments by Sharvin and Sharvin ${ }^{1}$ which experimentally demonstrated the existence of Aharonov-Bohm oscillations in disordered metal cylinders, ${ }^{2}$ the study of Aharonov-Bohm effects has brought to light many features of electrical transport which contradict the classical models. Foremost among them is that the diffusive propagation of the carriers does not cause the wave-function phase to be destroyed. ${ }^{3,4}$ Most of the observations of periodic and aperiodic ${ }^{5-7}$ quantum interference fluctuations $\Delta R$ of the magnetoresistance $R(H)$ in small metal loops and lone wires have been at length scales $L \simeq L_{\varphi}$; i.e., most of the carriers traverse the distance between voltage probes without randomization of the wave-function phase. In this coherent regime, ${ }^{8,9}$ the rms amplitude of the fluctuations in conductance is $\Delta G=C e^{2} / h$, where $C$ is a prefactor (of order 1) which depends upon the geometry of the sample. When $L_{\varphi} \gg L$, however, ${ }^{10,11}$ the resistance fluctuations become independent of length while the naive estimate of the conductance fluctuations diverges as $1 / L^{2}$. Moreover, it has been shown that the fluctuations can be observed in a device geometry for which classical physics would predict a null measurement. ${ }^{10,12}$ Indirect evidence for nonlocal behavior has also been seen in the correlation scales for random conductance fluctuations. ${ }^{13}$ In this work direct observations of nonlocal fluctuations are presented in detail. We demonstrate that the same $L_{\varphi}$ which describes the length dependence for the local resistance fluctuations accurately predicts the length dependence of nonlocal resistance fluctuations. In addition, we compare our results to the predictions of recent theories and find significant discrepancies with the analytical approaches ${ }^{11}$ but good agreement with numerical simulations. $^{14}$

Our measurements of resistance rely on the usual fourprobe methods. Two contacts $j$ and $k$ are used to source and sink current, and two others $m$ and $n$ are attached to a high-impedance meter that measures the voltage drop: $R_{m n, j k} \equiv\left(V_{m}-V_{n}\right) / I_{j \rightarrow k}$. The magnetic field depen- dence of the resistance in any such measurement can be decomposed into two parts, one symmetric and the other antisymmetric with respect to the reversal of the magnetic field. ${ }^{15}$ Upon decomposing the fluctuations $\Delta R=R$ $-\langle R\rangle$, Benoit et al. ${ }^{10}$ observed that the fluctuations in $R_{S}=\left[\left(R_{m n, j k}(\mathbf{H})+R_{m n, j k}(-\mathbf{H})\right] / 2\right.$, the symmetric component of $\Delta R$, and in $R_{A}=\left[R_{m n, j k}(\mathbf{H})\right.$ $\left.-R_{m n, j k}(-\mathbf{H})\right] / 2$, the antisymmetric component, scale very differently with length. A nearly universal method of characterizing the length dependence of the resistance fluctuations for any one-dimensional wire (which should eliminate all sample dependent parameters except the geometrical prefactors $C$ ) is as follows. The measured rms values of the magnetoresistance fluctuation amplitudes, $R_{S}$ and $R_{A}$, are normalized to the amplitude $\Delta R_{\varphi}=R_{\varphi}^{2} C e^{2} / h$ (which is approximately the total fluctuation amplitude expected for a wire segment of length $L_{\varphi}$ and classical resistance $R_{\varphi}$ ), and the distance between voltage probes is scaled by $L_{\varphi}$. For $L / L_{\varphi} \rightarrow 0$, $\Delta R / R_{\varphi} \simeq 0.37$ and is nearly independent of $L$, while for $L / L_{\varphi} \gg 1, \Delta R / R_{\varphi}$ grows in proportion to $\left(L / L_{\varphi}\right)^{1 / 2}$. This behavior has been observed in several samples, each with a different $L_{\varphi}$, with the results that all the normalized resistance fluctuations $\Delta R\left(L / L_{\varphi}\right) / \Delta R_{\varphi}$ fall on the same curve. ${ }^{10}$

The physical explanation for the observation of the length-independent behavior of the magnetoresistance fluctuations when $L_{\varphi} \gg L$ is that the carriers diffusing through the sample make excursions outside of the classical conduction path. On average, the currents caused by such excursions are zero (by definition of the classical conduction path), but in the coherent case, where the averages do not tell all of the story, such trajectories enclose magnetic flux and contribute to Aharonov-Bohm fluctuations in the conductance. ${ }^{16}$ The principal conceptual point to be understood is that on average the wave function for each carrier remains coherent for a time $\tau_{\varphi}$. During this time the carrier diffuses into an area $L_{\varphi}^{2}=D \tau_{\varphi}$, where $D$ is the classical diffusion coefficient. 


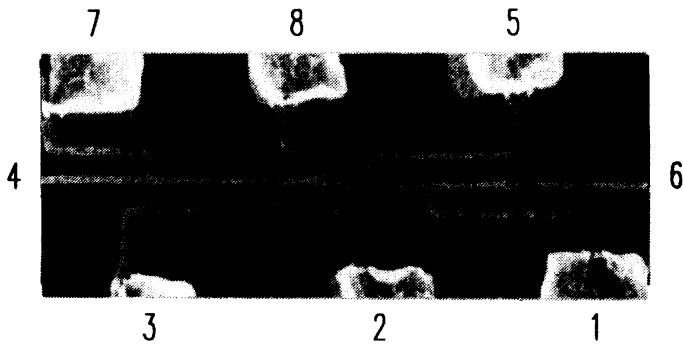

(a)

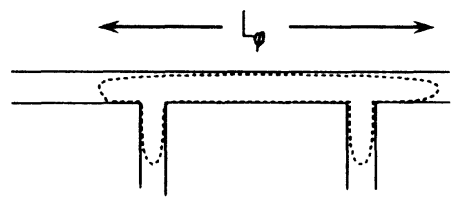

(b)

FIG. 1. (a) An electron microscope photograph of the $\mathrm{Sb}$ sample used in these experiments. The numbers labeling the leads are referred to in the text. (b) A schematic illustration of a four-probe sample. Typical regions (dotted lines) wherein the carriers retain phase coherence include some distance into the voltage probes, which contributes to nonlocal resistance fluctuations.

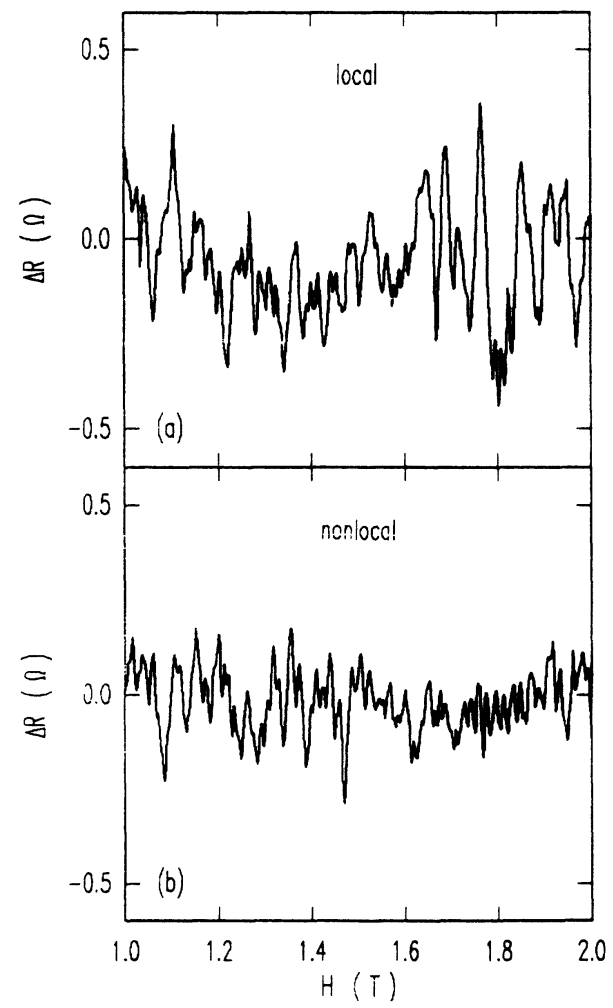

(We make the argument for two dimensions because, in these experiments, the resistance fluctuations result from interference of random trajectories which haphazardly enclose the fluxoids of the applied magnetic field and therefore lead to random Aharonov-Bohm oscillations, and one is concerned with areas in the discussion of Aharonov-Bohm effects. In the experiment, of course, the wave function is never outside of the wires which form the device.) Since the voltage probes [say, for instance, leads 8 and 3 of Fig. 1(a) in $R_{3,8 ; 4,6}$, the "local resistance"] are not phase randomizing reservoirs, they are generally of the same material and dimension as the classical conducting path (from lead 4 to lead 6), the carriers propagate into or beyond the probes while still retaining memory of the wave-function phase [see Fig. 1(b)]. This pervasiveness of the wave function is responsible for the observation ${ }^{10}$ that the nonlocal resistance as measured between leads 8 and 6 also fluctuates randomly when the current flows between leads 4 and 3. A typical record of both the local and nonlocal resistance fluctuations $\Delta R(H)$ as a function of magnetic field is displayed in Figs. 2(a) and 2(b). The corresponding autocorrelation functions are shown in Figs. 2(c) and 2(d). The sample in this case is an $\mathrm{Sb}$ wire $0.12 \mu \mathrm{m}$ wide and $0.08 \mu \mathrm{m}$ thick, and the probe spacing was $L=0.66 \mu \mathrm{m}$. The fluctuation amplitude is proportional to the current for small currents. The amplitude and field scale (determined from autocorrelation) of the resistance fluctuations are similar to that for the conventional measurement configuration

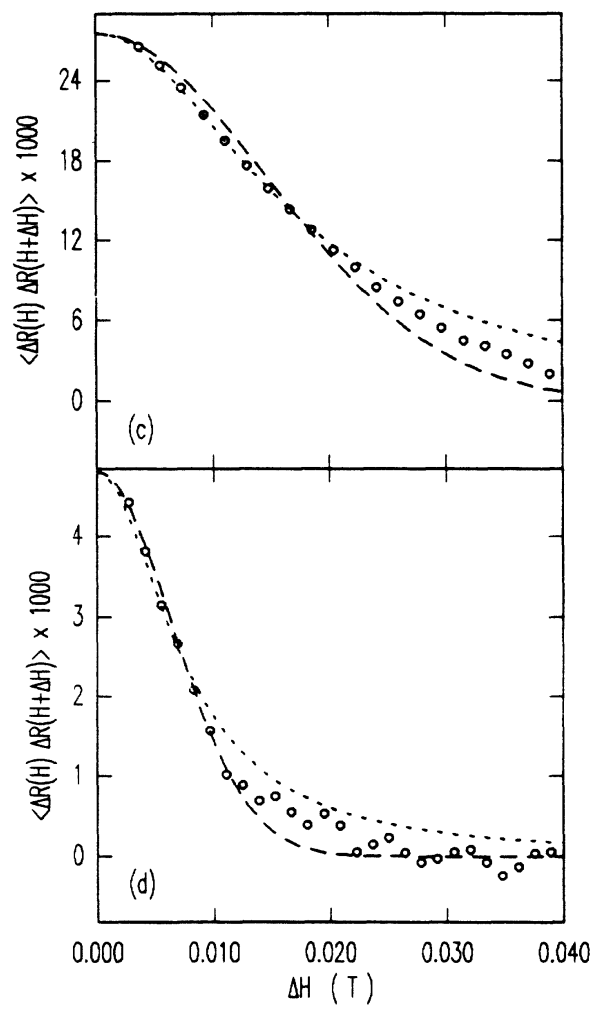

FIG. 2. (a) An example of reproducible magnetoresistance fluctuations in the local measurement $R_{8,3 ; 6,4}$ on the $0.66 \mu \mathrm{m}$ Sb wire. The sample temperature was $T=0.048 \mathrm{~K}$, and the drive current was $20 \mathrm{nA}$. (b) An example of the fluctuations in the nonlocal measurement $R_{8,6 ; 4,3}$ under the same conditions. (c) and (d) are autocorrelation functions (over the magnetic field range $-6<H<6 \mathrm{I}$ ) for the resistances in (a) and (b), respectively. The dashed and dotted lines illustrate, respectively, Gaussians and Lorentzian shapes with the same half-widths as the data from the experiments. 
[e.g., in Fig. 1(a), current from 4 to 6 and voltage drop measured between 3 and 8] when $L_{\varphi} \gg L_{3,8}$. Classical physics predict that, for homogeneous material, the voltage in the nonlocal measurement is zero [or rather $\sim \exp (-L / w)]$. We find in this transport measurement, then, direct evidence for the nonlocal character of the quantum-mechanical wave functions of the carriers. ${ }^{10}$

It is worth remarking that resistance fluctuations of similar character are observed in any measurement configuration where $L \lesssim L_{\varphi}$, regardless of whether the classical current path spans any of the voltage probes or not. ${ }^{10}$ This amplitude of the total fluctuation, when cast in the form of conductance, is $\Delta G \simeq e^{2} / h$, where one must be careful to notice that the effective length of the phase coherent sample is never less than $L_{\varphi \cdot}{ }^{10,11}$ It is this minimum size of the effective sample area which accounts for the $L$ independence of the amplitude of the observed fluctuations when $L<L_{\varphi}$.

We have studied the length dependence of the symmetric and antisymmetric parts of the nonlocal voltage fluctuations in the $\mathrm{Sb}$ sample [Fig. 1(a) and in two $\mathrm{Au}$ samples. $L_{\varphi}$ was determined from the weak localization effects in the magnetoresistance near $H=0$ of the $\mathrm{Sb}$ sample, and from the half-width of the autocorrelation function $C(\Delta H) \equiv\langle R(H) R(H+\Delta H)\rangle$ (where \langle\rangle is an average over a magnetic field range containing many correlation scales $B_{C}$ ) in segments of length $L \simeq L_{\varphi}$ in both the $\mathrm{Au}$ and $\mathrm{Sb}$ samples. The length $L$ that we use for analysis of the nonlocal measurements is defined to be the distance from the nearest voltage probe to the classical current path, and the separation of the midpoints of the voltage probes is used in the local measurements. From nonlocal measurements at different $L$, we obtained the dependence of $\Delta R^{\mathrm{NL}}$ on the ratio $L / L_{\varphi}$, and local measurements on the same wires yielded $\Delta R^{L}$. The symmetric and antisymmetric parts of the fluctuations were determined by lead switching experiments and by construction of $\Delta R_{S}$ and $\Delta R_{A}$ from a single trace about zero field. $^{15}$ The magnetoresistance traces were filtered digitally to remove signals outside of the range field scales expected for the conductance fluctuations, and the contribution to the measured resistance fluctuations from amplifier noise was removed by subtracting the noise power spectrum (estimated from "dummy" magnetoresistance traces at zero drive current) from the power spectrum of the fluctuations. From the resulting magnetoresistance curves, average fluctuation amplitudes were calculated. The results of these experiments for both the local and nonlocal resistance fluctuations are displayed in Figs. 3(a) and 3(b), respectively. In contrast to the length-independent behavior of the antisymmetric and symmetric (for $L<L_{\varphi}$ ) part of the local resistance fluctuations, ${ }^{10}$ both the symmetric part of the antisymmetric part of the nonlocal resistance fluctuations decay exponentially as $L / L_{\varphi}$ increases for all values of $L / L_{\varphi}$. The dot-dashed curve in Fig. 3(b) depicts the function $0.37 \exp \left(-a L / L_{\varphi}\right)$. The factor $a=1.2 \pm 0.3$ in the exponential is determined from a best fit to all of our data from several experiments, and it is consistent with recent theoretical calculations $(a=1.1 \pm 0.1){ }^{17}$ Since the data
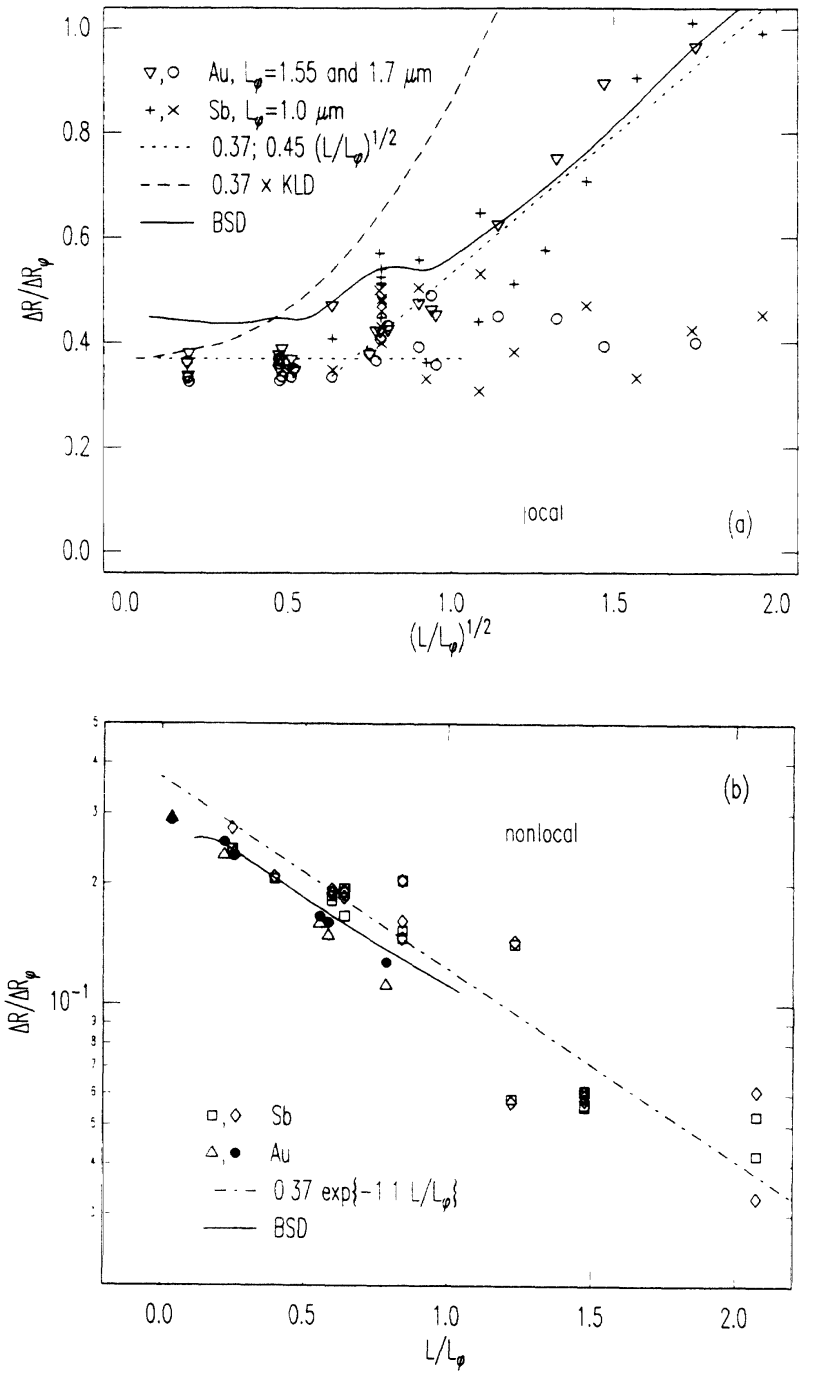

FIG. 3. (a) The dependence of the rms resistance fluctuations $\Delta R / \Delta R_{\varphi}$ on $L / L_{\varphi}$ averaged over the magnetic field range $-6<H<6 \mathrm{~T} . \Delta R_{S}$ from the Au wires $(\nabla)$ at $T=0.048 \mathrm{~K}$ and from the $\mathrm{Sb}$ sample $(+)$ are both independent of length when $L \rightarrow 0$, and they are $\propto \sqrt{L}$ when $L \gg L_{\varphi}$. The solid line is the result from the numerical simulations (Ref. 14), and the dashed line is the analytical theory (Ref. 11). The dotted lines indicate constant amplitude and the classical addition of fluctuations $(\propto \sqrt{L})$. In contrast, the antisymmetric components [O (Au) and $\times(\mathrm{Sb})]$ are essentially independent of length for all $L$. (b) The nonlocal fluctuations (both symmetric and antisymmetric components) from the same $\mathrm{Au}(\triangle$ and $O$ ) and $\mathrm{Sb}(\square$ and $\diamond)$ wires. The solid line is the result from the numerical simulations (Ref. 14), and the dash-dotted line is the fitted exponential decay described in the text.

include results from three different samples with different values of $L_{\varphi}$, we conclude that the appropriate scale for describing the decay of the nonlocal resistance fluctuations is $L / L_{\varphi}$ and not simply $L$. This is in accord with expectations. ${ }^{17}$ From classical diffusion, it is known that the amount of time spent in a dangling lead (the voltage 
probe is effectively dangling because it does not sink current) is proportional to the length of the lead. ${ }^{18}$ Since the electrons in the dangling lead lose phase coherence on length scale $L_{\varphi}$, only a fraction $e^{-L / L_{\varphi}}$ arrive at the voltage probe retaining phase memory. This is the source of the observed exponential decay.

There are theoretical results ${ }^{14}$ which suggest that, instead of being exponential, at zero temperature, the decay of $\Delta R^{\mathrm{NL}}$ should be power-law $\left[(\Delta R)^{2} \propto L^{-1}\right]$ with the restriction that (of course) the nonlocal fluctuations cannot be larger than those measured along the classical current paths. While there may be such an algebraic decay "beneath" the dominant exponential decay in Fig. 3 (b), the data appear to exclude scaling exponents greater than about 0.3 as $L / L_{\varphi} \rightarrow 0$. For $L / L_{\varphi}<1$, the data track this power law as well as they track the exponential. Our data, however, are certainly not consistent with a single power-law decay for $L / L_{\varphi}>1$.

The "local"" measurements [displayed in Fig. 3(a)] in which the voltage probes touch the classical current path were performed on the very same wires used for the nonlocal measurements. These measurements were decomposed into $\Delta R_{S}$ and $\Delta R_{A}$. Within the scatter, $\Delta R_{A}$ $(O, X)$ is independent of the separation of the probes as expected from analogy with classical Hall effects. The slight length dependence predicted in the theoretical calculations $^{11}$ is within the scatter of the data, and so, can neither be confirmed nor refuted. The symmetric part of the resistance fluctuation $\Delta R_{S}(\nabla,+)$ is essentially independent of $L$ as $L / L_{\varphi} \rightarrow 0$; the small predicted ${ }^{11}$ variation is within the scatter. As $L / L_{\varphi}$ increases well beyond $1, \Delta R_{S}$ grows approximately as $\left(L / L_{\varphi}\right)^{1 / 2}$. Both observations are in agreement with previous work. ${ }^{10}$ Both the local and the nonlocal resistance fluctuations extrapolate to $\Delta R / R_{\varphi}=0.37 \pm 0.1$ when $L=0$. For a given sample, the same value of $L_{\varphi}$ describes the local and nonlocal data. Moreover, these values of $L_{\varphi}$ are in agreement with $L_{\varphi}$ from weak localization (from the one $\mathrm{Sb}$ wire which exhibits it) and the characteristic field scale $B_{C}$ obtained for large $L / L_{\varphi}$.

The results of numerical simulations ${ }^{14}$ of the fluctuations in multiple-probe samples are also displayed in Fig. 3(a) (solid lines). The simulation calculated the total fluctuation amplitude at zero magnetic field. To compare the simulations with the data in Fig. 3(a), we have divided the calculated $\Delta R / R_{\varphi}$ by $\sqrt{2}$ to remove the Cooperon contribution ${ }^{9}$ (since in our experiments $H \gg h / 2 L_{\varphi}^{2}$, the Cooperon contribution is negligible) and multiplied it by 2 in order to account for spin degeneracy. Using $R_{\varphi}=0.49 h / e^{2}$, we have the normalization $\Delta R_{\varphi}=\left(e^{2} / h\right) R_{\varphi}^{2}=0.24 h / e^{2}$. This obtains excellent quantitative agreement with the data over the entire range of $L / L_{\varphi}$ with no adjustable parameters. This quantitative agreement between experiment and numerical simulations constitutes strong evidence in support of the universal scaling of the length dependence of the voltage fluctuations in any phase coherent wire.

In the simulations, $L_{\varphi}$ is set by the placement of fixed reservoirs at various points on the sample (specifically they are at the ends of the voltage probes). The correspondence between this model and samples used in the experiments is unclear, because we expect phase destruction to occur randomly throughout the samples. The analytical theory includes the phase destruction as an ad hoc lifetime $\tau_{\varphi}$ for the propagators in the theory, which seems more compatible with this expectation. The prediction from the analytical theory by Kane, Lee, and DiVincenzo ${ }^{11}$ [their Eq. (C8)] which is valid for $L_{T} \equiv\left(h D / k_{B} T\right)^{1 / 2} \rightarrow \infty$ is displayed in Fig. 3(a) as a dashed line. Although $L_{T} \rightarrow \infty$ does not describe the conditions under which the data were recorded (for Fig. 3, $L_{\varphi} \simeq L_{T}$ ), allowing finite $L_{T}$ cannot repair the discrepancy. ${ }^{19}$ Data from lower temperature $(T=0.010$ $\mathrm{K})$ and lower drive current $(I=10 \mathrm{nA})$, where $L_{T}$ is even longer, have the same scaling as a function of $L / L_{\varphi}$ as those in Fig. 3. To fix the discrepancy $L_{T}$ must be substantially less than $L_{\varphi}$ (one needs $L_{T}<0.5 L_{\varphi}$ ). From analysis of $B_{C}$ we find that, for most of the data, $L_{\varphi}<L_{T}$, and this agrees with estimates made from the resistivity and with direct measurements of the correlation energy (which governs $L_{T}$ ) via the $I-V$ curves from the wires. ${ }^{20}$ In principle, the presence of dangling leads (e.g., leads 2 and 8 in the resistance $R_{3,5 ; 4,6}$ ) between the voltage probes offers more phase space for the carriers, and so these extra probes can effectively lengthen the sample. From the experiment, we do not find a significant difference between the measurements with and without the dangling probes, and all data are plotted on the same graph. The difference in the theoretical model caused by the dangling probes is around $20 \%$, which is about the scatter in our data.

In plotting the data, we have assumed the geometrical prefactor $^{9}$ is $C=0.73$. At $L=0$, however, Kane, Lee, and DiVincenzo have the symmetric part of the normalized voltage fluctuation equal to 1 with $C=1$ in $\Delta R_{\varphi}$, which is too high by a factor of $\simeq 2.7$, and we have divided by this factor in Fig. 3(a). Blindly normalizing the theoretical curve at $L=L_{\varphi}$, we find that it gives the same $L \rightarrow 0$ asymptote as the experiment, but inserting the prefactor $C=0.73$ into the theory makes the disparity even worse than a factor of 2.7. More importantly, the quantitative agreement between the length scaling in analytical theory with that in the experiment and numerical simulations is very poor, although the trends are similar. In order for the theory to be placed in quantitative agreement with the experimental data, the experimental values of $L_{\varphi}$ must be increased by a factor $\simeq 2.5$. This forces the lowering of the $L \rightarrow 0$ asymptote because it increases $\Delta R_{\varphi}$ by the square of the factor, so that this procedure results in $\Delta R / \Delta R_{\varphi} \simeq 0.06$ for the data-nearly an order of magnitude lower than the value 0.4 known from numerous experiments in our laboratory. ${ }^{10}$ Arbitrarily increasing the theoretical $L / L_{\varphi}$ by a factor of about 2.5, without changing the $L=0$ asymptote, produces excellent agreement with the data. The choice to compare with the calculations by Kane, Lee, and DiVincenzo is one of convenience, and comparisons with other versions of the analytical theory ${ }^{11}$ arrive at the same difficulties, but the other versions do obtain a prefactor that is closer to the observed value. 
The crucial parameter in the test of scaling of the resistance fluctuation amplitude is $L_{\varphi}$. One way to determine $L_{\varphi}$ is to study the correlation field $B_{C}=z h / e L_{\varphi} w$, where $z$ is a numerical constant. In the two-probe theory, ${ }^{21} z$ is between 0.42 (when $L_{\varphi} \ll L_{T}$ ) and 0.95 (when $L_{\varphi}>L_{T}$ ) that can be evaluated theoretically for a given sample and a given ratio of $L_{\varphi}$ to the thermal diffusion length $\left.L_{T}\right){ }^{21}$ This argument has been worked out only for the case of two-probe measurements, and the corresponding arguments for four-probe measurements are only now being formulated. ${ }^{22}$ Our data clearly indicate that the shape of the correlation function and the inferred $B_{C}$ depend on the particular probe configuration. From Fig. 2(c) one sees that, in the local measurement, the correlation function is wider, and that it is more nearly approximated by a Lorentzian curve than by a Gaussian curve. From Fig. 2(d), we see that the opposite is true for the nonlocal measurement. [We do not suggest that a Gaussian or Lorentzian is an accurate description of the functional form of $C(\Delta H)$; we only use these as familiar examples of short- and long-range correlations.] These results are representative of our analysis of more than 100 local and nonlocal magnetoresistance measurements. In some cases the distinction in functional form is even more exaggerated: for the very long wires the $C(\Delta H)$ has a longer tail than a Lorentzian of the appropriate halfwidth. When $L<L_{\varphi}$, however, there is frequently an intermediate shape [for instance, in Fig. 2(c), the tail is not as long as the Lorentzian's]. In Fig. 4(a), the $B_{C}$ obtained from the total fluctuation $\Delta R(H)$ for a fraction of our magnetoresistance data are displayed. For convenience, the values from the nonlocal measurements are plotted at $-L$. The data demonstrate that $B_{C}$ and $C(\Delta H)$ depend on the measurement configuration in a rather dramatic way. The $B_{C}$ for a particular line segment is always larger in the local $(+L)$ measurement, than in the nonlocal $(-L)$ measurement.

The physical reason for the difference between the local autocorrelation function $C^{\mathrm{L}}(\Delta H)$ and the nonlocal autocorrelation function $C^{\mathrm{NL}}(\Delta H)$ is that different sets of paths contribute to the two different sets of resistance fluctuations. In particular, short-range $e^{23}(<L)$ loops of trajectories add together to generate a large fraction of $\Delta R^{\mathrm{L}}$ [see the right-hand inset to Fig. 4(b)]. In contrast in the nonlocal measurements (see the left-hand inset), only long $(\simeq L)$ loops matter. The arbitrarily short paths in the local measurements generate the long tail on $C^{\mathrm{L}}(\Delta H)$ since for the $k$ th individual loop, $B_{C} \sim 1 / L_{k}^{2}$ (where $L_{k}$ is the diameter of the loop) can be arbitrarily large. The absence of paths shorter than $L$ in the nonlocal measurements provides a hard cutoff for the range of $C^{\mathrm{NL}}(\Delta H)$. This dichotomy is apparent in the experimental data. At least for longer $L, C^{\mathrm{NL}}(\Delta H)$ is essentially Gaussian in shape [similar to Fig. 2(d)]. On the other hand, the tails on $C^{\mathrm{L}}(\Delta H)$ are better approximated by a Lorentzian curve, or for $L>1.6 \mu \mathrm{m}$ by a function with an even slower rolloff than $1 /(\Delta H)^{2}$.

We can compare our results to the theoretical prediction for $C(\Delta H)$ from a four-probe resistor. The model is a four-probe wire where the probes are the same width as the current-carrying channel, and the corners between the probe and the channels are square [i.e., Fig. 1(b)]. The analysis below therefore neglects the wide (twodimensional) regions that connect the narrow channels to the wire-bonding pads. This neglect is probably a good approximation for all probes except 2 and 8 , because with these exceptions, the two-dimensional regions are more than $2 L_{\varphi}$ from the measured segment. For pads 2 and 8 , the distance is about $L_{\varphi}$ and carriers that emerge into the wide regions have much more area to roam so that, for a given $L_{\varphi}$, there will be a contribution to $B_{C} \propto 1 / L_{\varphi}^{2}$ (in-
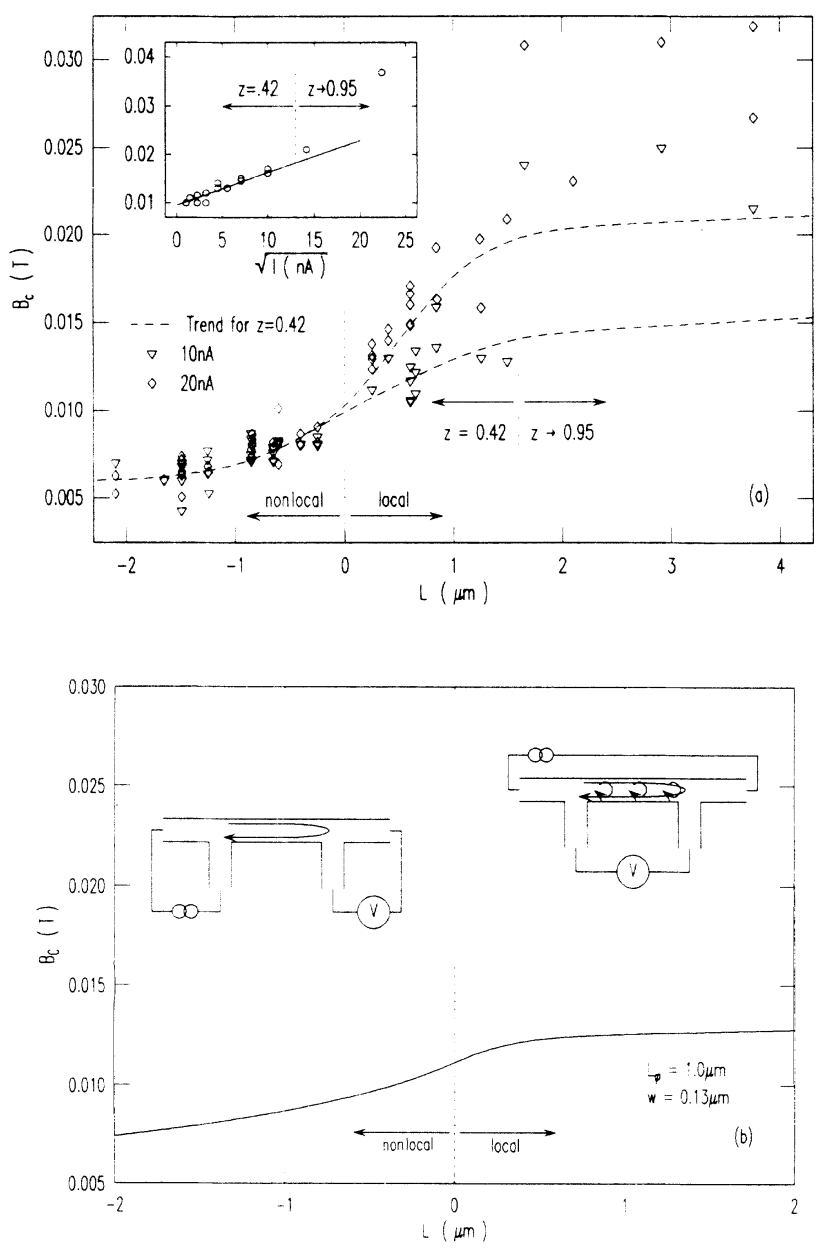

FIG. 4. (a) Correlation field scales from various line segments. The results from local measurements are plotted with positive $L$ and the nonlocal results with negative $L(L \rightarrow-L)$. Different symbols refer to different drive currents, and the dashed lines illustrate the $L$ dependence expected from the four-probe model (Ref. 22) when $z=0.42$. The inset illustrates the increase of $B_{C}$ as current increases at $T=0.01 \mathrm{~K}$ and the dashed line depicts the form expected from voltage averaging. (b) $B_{C}$ as a function of $L$ from the four-probe model (Ref. 22) plotted in the same manner as the experimental data. Inset on the left is an illustration of the kind of trajectories that contribute to fluctuations in the nonlocal measurements, and on the right side a similar picture for the local measurement (Ref. 23). 
stead of $1 / L_{\varphi} w$ as in the one-dimensional channels) and the total $B_{C}$ will be smaller. We estimate that, since the fraction of carriers that make such excursions and return is $<1 / e^{2}$, the error caused by ignoring the pads is no worse than $15 \%$ for these two probes and a good deal better for the others. From the theoretical model ${ }^{22}$ we have calculated the variation of $B_{C}$ with $L / L_{\varphi}$ [Fig. 4(b)] for the case of $L_{T} \rightarrow \infty$. The trend found in the experiment is reproduced here by the model. For a particular $L_{\varphi}$, the correlation field is smaller in the nonlocal measurement than in the local measurement, and there is a smooth crossover between the two regimes. The calculation was done for a particular width and $L_{\varphi}$, whereas $w$ (and possibly even $L_{\varphi}$ ) in the experiments vary from segment to segment. It is therefore not too surprising that we do not obtain precise quantitative agreement with the experiment. We emphasize, however, that both the theory and experiment demonstrate a significant length dependence to the measured $B_{C}$, for a constant $L_{\varphi}$, as the nonlocal contributions to the fluctuations become more important $\left(L<L_{\varphi}\right)$.

The above model is strictly linear response, so there is no parameter corresponding to current drive. Two current drives were used for the data in Fig. 4(a), and in the nonlocal $(-L)$ measurements, $B_{C}$ is independent of drive, but it depends rather strongly on it in the local $(+L)$ measurements. This difference between the nonlocal and local data can be qualitatively understood by allowing for the possibility of voltage averaging in the local measurements. There is a finite electric field along the measured segment in the local measurement, and the carriers therefore have an energy spread and a corresponding "effective temperature" $T_{\text {eff }} \simeq \mathrm{eV} / k_{B}$ which leads to voltage averaging and hence to a decrease in $L_{T}$. Perhaps, at very high currents, this could eventually lead to a decrease in $L_{\varphi}$, but we doubt that this occurs in any of the experiments here. The decrease in $L_{T}$ is apparent from the inset to Fig. 4(a), which displays $B_{C}$ from data recorded at other temperatures. Plotting $B_{C}$ versus $\sqrt{I}$,

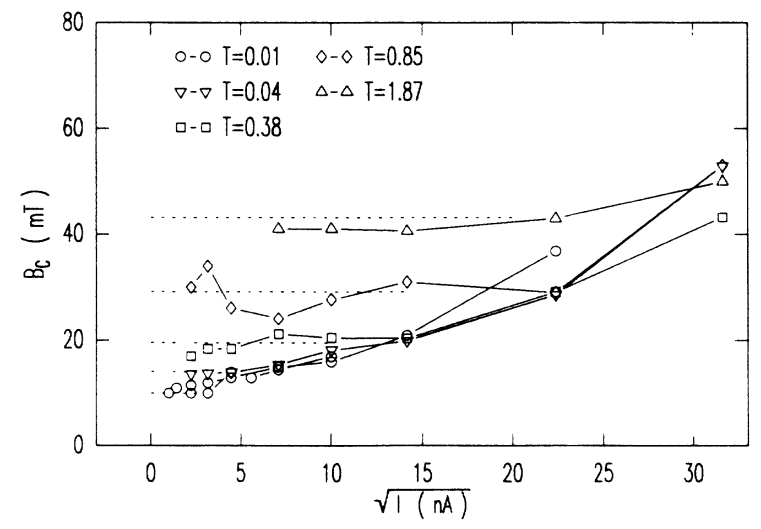

FIG. 5. The dependence of $B_{C}$ on drive current from a series of local measurements on the $0.66 \mu \mathrm{m} \mathrm{Sb}$ wire. Data obtained at several different temperatures are displayed, and for each temperature the zero current asymptote (dotted lines) agrees with a scaling $B_{C}(T, I=0) \simeq \sqrt{T}$. we find a linear dependence for $I<100 \mathrm{nA}$, and then a faster increase. The effective phase coherence length determining the correlation scale is the lesser of $L_{\varphi}$ and $L_{T}$. For the low-temperature data in Fig. $5(\circ, T=0.01$ $\mathrm{K}), L=0.66 \mu \mathrm{m}$ and $R=49 \Omega$ so that at $I=100 \mathrm{nA}$ the voltage bias is $5 \mu \mathrm{V}$, which exceeds the lattice temperature $(\simeq 1 \mu \mathrm{V})$. Making a modest extension of existing analysis, ${ }^{9,17}$ we suppose that if the effective phase relaxation rate is the sum of the voltage averaging "rate" and $\tau_{\varphi}$, then $B_{C}$ will grow as $1 / L_{T} \propto \sqrt{T_{\text {eff }} \propto \sqrt{I}}$. We assume that this accounts for the observed dependence of $B_{C}$ on $I$. In addition, the breakpoint where $B_{C}$ departs from $\sqrt{I}$ is near the voltage bias where $B_{C}(L)$ grows anomalously for the local measurements. We therefore attribute the very large values of $B_{C}$ in Fig. 4(a) at large $L$ to the crossover from the limit $L \ll L_{T}$ (where $z=0.42$ ) towards the opposite limit (where $z=0.95$ ). ${ }^{21}$ If the limit were not exceeded, we suppose that $B_{C}(L)$ would be much flatter at large $L$ as suggested by the model and illustrated by the dashed lines in Fig. 4(a). At successively higher temperatures, $B_{C}(I)$ saturates at higher values as $I \rightarrow 0$. The saturation levels are consistent with $B_{C}(0) \propto \sqrt{T}$ as illustrated by the dotted lines. In this sample $^{24} L_{\varphi}$ decreases with increasing temperature at least as fast as $L_{T}$, so that for $I \rightarrow 0$ at any temperature, we expect that $L_{T}$ always exceeds $L_{\varphi}$, and $z=0.42$.

A similar difference in $C(\Delta H)$ occurs between the symmetric (S) and antisymmetric (AS) components of a particular local measurement. The antisymmetric components of the resistance are generated mainly in the probes attached to the sample where the average voltage is zero. In contrast, the symmetric component is accumulated all along the current channel where the bulk of the voltage is dropped. In this and other ${ }^{25}$ experiments, we find that, quite generally, $B_{C}^{\mathrm{AS}}$ is smaller than $B_{C}^{\mathrm{S}}$.

Another potential source of error in Fig. 3 is the wire width. It is known, from tunneling spectroscopy, ${ }^{26}$ that thin layers of "metallic" atoms exhibit gaps at the Fermi level; i.e., they do not conduct at $T$ less than the gap energy. This is true up to $\gtrsim 20$ layers for the semimetal Sb when it is deposited commensurately onto a cleaved GaAs surface. The size of the gap presumably depends on the local environment, which leads us to suspect that there is an uncertainty of $\lesssim 10 \mathrm{~nm}$ in our estimates of the effective conducting widths of the $\mathrm{Sb}$ wires. The interface between the $\mathrm{Sb}$ and the vacuum is likely to be very different from the interface with the $\mathrm{Si}_{3} \mathrm{~N}_{4}$ substrate which has its own (incommensurate) lattice constant and gap, and we expect that the nonconducting regions along the sides of the wire are considerably less than the thickness observed for commensurate overlayers on the (110) surface of cleaved GaAs. A similar (albeit much smaller) uncertainty might be present in the Au samples. In principle we have yet another method of obtaining the effective widths of the wires. The classical magnetoresistance is proportional to $\left(\omega_{C} \tau\right)^{2}$, and from the rather large $(10 \%)$ quadratic field dependences in the Sb samples, we have extracted the "conductivities" $\sigma_{k}$ for the individual line segments from $R(H)$, and these are listed in Table I. There is scatter of about $25 \%$ among the various sam- 
TABLE I. Wire segment dimensions and classical conductivities (inferred from classical parabolic magnetoresistance) for the various $\mathrm{Sb}$ wire segments.

\begin{tabular}{lccc}
\hline \hline $\begin{array}{c}\text { Voltage taps } \\
\text { [see Fig. 1(a)] }\end{array}$ & $\begin{array}{c}\text { Length } \\
(\mu \mathrm{m})\end{array}$ & $\begin{array}{c}\text { Width } \\
(\mu \mathrm{m})\end{array}$ & $\begin{array}{c}\sigma \\
{\left[(\mu \Omega \mathrm{m})^{-1}\right]}\end{array}$ \\
\hline 2,8 & 0.25 & 0.164 & $0.96 \pm 0.02$ \\
2,5 & 0.45 & 0.125 & $0.75 \pm 0.02$ \\
8,3 & 0.66 & 0.125 & $0.88 \pm 0.01$ \\
5,1 & 0.89 & 0.102 & $0.83 \pm 0.01$ \\
7,3 & 1.79 & 0.098 & $0.71 \pm 0.01$ \\
\hline \hline
\end{tabular}

ples. Attempts to use these to obtain the true electrical widths of the samples have introduced so much scatter in $\Delta R\left(L / L_{\varphi}\right)$ that we could say nothing about the scaling dependence using this method. In part, the reason for this problem may be that the thickness of the wire segments is not uniform, and of course, there is the innate fallacy $^{27}$ of attempting to define a local conductivity on length scales shorter than many $L_{\varphi}$. As a result, we are forced to use the widths measured from the electron microscope photographs of the samples, and resign ourselves to the large error bars on the parameter $w$.

From the foregoing analysis, especially the universal scaling of both local and nonlocal resistance fluctuations with a value of $L_{\varphi}$ corroborated by weak localization and the magnetic correlation scale, we are confident that the errors from estimating $w$ and $L_{\varphi}$ are small enough to rule out agreement with the analytic theory for scaling $\Delta R\left(L / L_{\varphi}\right)$ which would require distending the abscissa of Fig. 3(a) by more than a factor of 2 . It would be very surprising if the $L_{\varphi}$ that yields agreement with the exponential decay of the nonlocal fluctuations were not appropriate to describe the "local" fluctuations. There appears then to be a flaw in the theory: it correctly predicts the decay of the fluctuations measured when the voltage probes do not touch the classical current path, but it fails to predict the scale on which the fluctuations accumulate when the current flows past the voltage probes. In the limit $L_{T} \ll L_{\varphi}$, it is appropriate to scale ${ }^{21}$ the $L_{\varphi}$ inferred from the field correlation scale by a factor 2.2 , but this limit is never achieved in the experiments displayed in Figs. 3(a) and 3(b). The closest approach is $L_{T} / L_{\varphi}=0.8$ for the data at $0.048 \mathrm{~K}$; for the Au samples, $L_{T}>L_{\varphi}$, and for the data at lower temperatures the ratio exceeds 1 in all samples.

Even if we ignore all of the experimental evidence for $L_{\varphi}$ and allow it to float as a free parameter, we still cannot reconcile the analytical theory with the data. Adjusting $L_{\varphi}$ means changing $\Delta R_{\varphi} \sim L_{\varphi}^{2}$. Forcing $\Delta R_{S}\left(L / L_{\varphi}\right)$ to fit the theoretical curve, forces a corresponding adjustment of $\Delta R_{\varphi}$ which, in turn, reduces the asymptote for $\Delta R_{e}(L \rightarrow 0) / \Delta R_{\varphi}$ to about an order of magnitude below that predicted asymptote.
Having (by an admittedly stony path) concluded that the analytical calculations are a poor description of the scaling of the local resistance fluctuations while the numerical calculations are much better, we must ask why. Prima facie, the data in Fig. 3(a) imply that the fixed phase reservoirs used in the numerical calculation are a better model for the real samples than the (ad hoc) homogeneous inelastic scattering invoked in the analytical theory. This is plausible because we study a particular sample with a particular impurity potential and not the ensemble which underlies the analytical theory. Each sample contains fixed grain boundaries and impurities, and these provide for strong local potentials, and the electron-electron or electron-phonon scattering may be stronger in the vicinity of such potentials. (This is in the spirit of the interaction effects in weak localization, where the potential fluctuations force the electrons to spend more time in close proximity than free carriers do.) While this is certainly not the only explanation for the discrepancy between our data and the analytical theory, we have not seen a better one to date.

In summary, we recall that experiments have provided strong evidence in support of the universal scaling of the length dependence of the nonlocal and local resistance fluctuations in small phase coherent wires. The length independence of the local voltage fluctuations (for $L_{\varphi}>L$ ) results from the extension of the carrier wave functions into all regions of the sample where the classical current would be exactly zero. Using the phase coherence length determined from weak localization and the autocorrelation function of the resistance fluctuations in long segments $\left(L>L_{\varphi}\right)$, we find excellent agreement with numerical simulations for the scaling of the local fluctuations with probe separation, as well as agreement with recent analytic calculations for the exponential decay of the nonlocal fluctuations. The analytic theory for the length dependence of the local fluctuations appears to have a length scale which differs from the experiment by a factor of $\simeq 2.5$. We suggest that this difference between the analytic and numerical theories may be due to the precise details of how inelastic scattering is incorporated, and we note that in real samples the destruction of phase coherence appears to occur at spatially fixed points. The measured correlation field scales $B_{C}$ also exhibit a length dependence and significant modifications to the standard relationship between $L_{\varphi}$ and $B_{C}$ must be made when nonlocal effects contribute significantly to the measurement. In addition, the observed voltage dependence of $B_{C}$ is consistent with the results from a four-probe model if the "heating" of the electrons by the drive current is accounted for in a simple way.

We gratefully acknowledge helpful discussions with $\mathbf{B}$. Al'tshuler, H. Baranger, M. Büttiker, V. Chandrasekhar, D. DiVincenzo, Y. Gefen, S. Hershfield, Y. Imry. C. Kane, P. Lee, S. Maekawa, P. Santhanam, and D. Stone. 
"Present address: Department of Physics and Astronomy, University of Maryland, College Park, MD 20742-4111.

tPermanent address: Centre de Recherches sur les Très Basses Températures, Centre National de la Recherche Scientifique, 38042 Grenoble, France

${ }^{1}$ D. Yu. Sharvin and Yu. V. Sharvin, Pis'ma Zh. Eksp. Teor. Fiz. 34, 285 (1981) [JETP Lett. 34, 272 (1981)].

${ }^{2}$ B. L. Al'tshuler, A. G. Aronov, and B. Z. Spivak, Pis'ma Zh. Eksp. Teor. Fiz. 33, 101 (1981) [JETP Lett. 33, 101 (1981)].

${ }^{3}$ Y. Gefen, Y. Imry. and M. Ya. Azbel, Surf. Sci. 142, 203 (1984); Phys. Rev. Lett. 52, 129 (1984); M. Bütiker, Y. Imry, R. Landauer, and S. Pinhas, Phys. Rev. B 31, 6207 (1985).

${ }^{4}$ R. Landauer, Philos. Mag. 21, 863 (1970).

${ }^{5}$ R. A. Webb, S. Washburn, C. P. Umbach, and R. B. Laibowitz, Phys. Rev. Lett. 54, 2696 (1985); V. Chandrasekhar, M. J. Rooks, S. Wind, and D. E. Prober, Phys. Rev. Lett. 55, 1610 (1985); S. Datta et al., ibid. 55, 2344 (1985).

${ }^{6}$ R. A. Webb, S. Washburn, C. P. Umbach, and R. B. Laibowitz, in Localization, Interaction, and Transport Phenomena in Impure Metals, edited by G. Bergmann, Y. Bruynseraede, and B. Kramer (Springer, Heidelberg, 1985); C. P. Umbach, S. Washburn, R. B. Laibowitz, and R. A. Webb, Phys. Rev. B 30, 4048 (1984).

${ }^{7}$ For reviews, see Y. Imry, in Directions in Condensed Matter Physics, edited by G. Grinstein and E. Mazenko (World Publishing, Singapore, 1986), p. 101; S. Washburn and R. A. Webb, Adv. Phys. 35, 375 (1986); A. G. Aronov and Yu. V. Sharvin, Rev. Mod. Phys. 53, 755 (1987).

${ }^{8}$ B. L. Al'tshuler, Pis'ma Zh. Eksp. Teor. Fiz. 41, 530 (1985) [JETP Lett. 41, 648 (1985)].

${ }^{9}$ P. A. Lee, A. D. Stone, and H. Fukuyama, Phys. Rev. B 35, 1039 (1987).

${ }^{10}$ A. D. Benoit, C. P. Umbach, R. B. Laibowitz, and R. A. Webb, Phys. Rev. Lett. 58, 2343 (1987); W. J. Skocpol, P. M. Mankiewich, R. E. Howard, L. D. Jackel, D. M. Tennant, and A. D. Stone, ibid. 58, 2347 (1987).

${ }^{11}$ S. Maekawa, Y. Isawa, H. Ebisawa, J. Phys. Soc. Jpn. 56, 25 (1987); Y. Isawa, H. Ebisawa, and S. Mackawa, in Anderson Localization, edited by T. Ando and H. Fukuyama (Springer, Heidelberg, 1988); M. Büttiker, Phys. Rev. B 335, 4123
(1987); A. Yu. Zyuzin and B. Z. Spivak, Zh. Eksp. Teor. Fiz. 93, 994 (1987) [Sov. Phys.-JETP 66, 560 (1987)]; S. Hershfield and V. Ambegaokar, Phys. Rev. B 38, 7909 (1988); C. L. Kane, P. A. Lee, D. P. DiVincenzo, ibid. 38, 2995 (1988).

${ }^{12}$ C. P. Umbach, P. Santhanam, C. van Haesendonck, R. A. Webb, Appl. Phys. Lett. 50, 1289 (1987).

${ }^{13}$ W. J. Skocpol, P. M. Mankiewich, R. E. Howard, L. D. Jackel, D. M. Tennant, and A. D. Stone, Phys. Rev. Lett. 56, 2865 (1986).

${ }^{14}$ H. Baranger, A. D. Stone, D. P. DiVincenzo, Phys. Rev. B 37, 6521 (1988).

${ }^{15}$ M. Büttiker, Phys. Rev. Lett. 57, 1761 (1986); A. Benoit, S. Washburn, C. P. Umbach, R. B. Laibowitz, and R. A. Webb, ibid. 57, 1765 (1986).

${ }^{16}$ A. D. Stone, Phys. Rev. Lett. 54, 2692 (1985).

${ }^{17}$ D. P. DiVincenzo and C. L. Kane, Phys. Rev. B 38, 3006 (1988).

${ }^{18}$ Compare I. Goldhirsch and Y. Gefen, Phys. Rev. A 33, 2583 (1986).

${ }^{19}$ S. Hershfield, Ann. Phys. (N.Y.) 196, 12 (1989).

${ }^{20}$ R. A. Webb, S. Washburn, and C. P. Umbach, Phys. Rev. B 37, 8455 (1988) (unpublished).

${ }^{21}$ C. W. J. Beenakker and H. van Houten, Phys. Rev. B 37, 6544 (1988).

${ }^{22}$ V. Chandrasekhar, P. Santhanam, and D. E. Prober (unpublished).

${ }^{23}$ B. Al'tshuler (unpublished).

${ }^{24}$ F. P. Milliken, S. Washburn, C. P. Umbach, R. B. Laibowitz, and R. A. Webb, Phys. Rev. B 36, 4465 (1987); F. P. Milliken, S. Washburn, M. McCord, M. J. Brady, and R. A. Webb (unpublished).

${ }^{25}$ W. Hansen, T. P. Smith, D. P. DiVincenzo, K. Y. Lee, Y. H. Lee, and R. Cheung, in Proceedings of a Conference on Electronic Properties of Two-dimensional Systems, Grenoble, 1989 (unpublished).

${ }^{26}$ C. K. Shih, R. M. Feenstra, and P. Mårtenssen, J. Vac. Sci. (to be published).

${ }^{27}$ C. L. Kane, R. A. Serota, and P. A. Lee, Phys. Rev. B 37, 6701 (1988) 


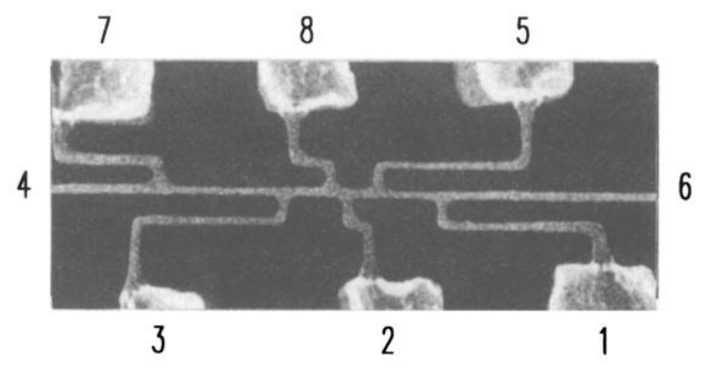

(a)

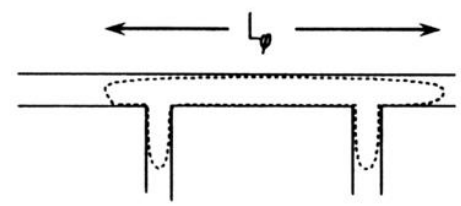

(b)

FIG. 1. (a) An electron microscope photograph of the Sb sample used in these experiments. The numbers labeling the leads are referred to in the text. (b) A schematic illustration of a four-probe sample. Typical regions (dotted lines) wherein the carriers retain phase coherence include some distance into the voltage probes, which contributes to nonlocal resistance fluctuations. 